Trauma Berufskrankh 2007 - 9[Suppl 3]:

S351-S358

DOI 10.1007/s10039-007-1301-9

Online publiziert: 26. Oktober 2007

(c) Springer Medizin Verlag 2007

\author{
S. Özkir · P. Droste - V. Echtermeyer \\ Unfallchirurgische Klinik, Minden
}

\title{
Wann wird zementiert?
}

Ein besonderes Ziel in der Orthopädie und Unfallchirurgie war es immer:

- die Beweglichkeit der arthrotisch, posttraumatisch und postarthritisch bedingten Versteifungen im Hüftgelenk wiederherzustellen,

- die Patienten von den z. T. erheblichen Schmerzen zu erlösen und

- die damit verbundene Verminderung der Lebensqualität wieder zu mildern.

Die Implantation der Hüftendoprothese zählt heutzutage zu den bemerkenswertesten Fortschritten des letzten Jahrhunderts in der Medizin. Seit der klinischen Etablierung dieses Verfahrens vor über 40 Jahren wurden weltweit über 6 Mio. Eingriffe durchgeführt $[8,9]$. Dies entspricht weltweit etwa einem jährlichen Gesamtumsatz von 620 Mio. US-Dollar [77].

Durch die immer weiter fortschreitende Entwicklung in der Hüftgelenkendoprothetik nimmt diese Behandlungsform bei jährlich etwa 500.000 implantierten Hüftendoprothesen in Europa eine zentrale Rolle in der unfallchirurgischen und orthopädischen Tätigkeit ein [68, 77]. In Deutschland werden jährlich etwa 200.00o Hüftendoprothesenoperationen mit einer Vielzahl von Prothesenmodellen durchgeführt [55], von denen etwa $10 \%$ zementiert werden [50]. Die Zahl der Wechseloperationen in Deutschland liegt derzeit zwischen 5 und 10\% [55].

\section{Dauerhafte Prothesenimplantation}

Die Implantation einer Hüftgelenkprothese ist heute ein erfolgreiches und effektives Verfahren in der Gelenkchirurgie. Inzwischen ist weltweit eine große Auswahl von Hüftprothesensystemen verfügbar, wobei sich grundsätzlich zementierte und unzementierte Systeme unterscheiden lassen.
Ziel beim künstlichen Hüftgelenkersatz sind die dauerhafte Fixation und schmerzfreie Funktion der Prothesenkomponenten im Knochen und die Minimierung des Abriebs zwischen den Gleitpartnern Kopf und Pfanne [34, 45, 46, 87, 88]. Die Funktionsfähigkeit der Kunstgelenke ist jedoch unabhängig von der Implantationstechnik limitiert, denn es sollte nicht übersehen werden, dass die Problematik der aseptischen Lockerung, trotz aller uns heute zur Verfügung stehenden Implantate, im Hinblick auf die Langzeitergebnisse nicht gelöst ist und weiterhin in Kombination mit dem periprothetischen Knochenverlust die häufigste Spätkomplikation darstellt $[49,67]$. Dies betrifft sowohl den Schaft als auch die Pfanne, unabhängig von zementierter oder zementfreier Implantation. In groß angelegten Studien wurden in der primären Hüftendoprothetik generelle Lockerungsraten von $4-10 \%$ nach 10 Jahren Standzeit beschrieben $[1,26]$. Im Bestreben, die langlebigste Implantatkombination zu erreichen, sind in den letzten Jahrzehnten eine Vielzahl verschiedener Pfannen- und Schaftdesigns entwickelt worden, aus welchen sich über 20 Kombinationsmöglichkeiten ergeben.

Abgesehen von wenigen Ausnahmen (z. B. verbesserte Zementiertechnik, Entwicklung neuer PMMA-Knochenzemente) haben die meisten Neuerungen in der Hüftendoprothetik bis heute zu keiner gesicherten Verbesserung der Standzeiten geführt. Entsprechend der Forderungen des „National Institute of Health“ [61] muss von einem Prothesensystem eine Überdauerungsrate von 95\% nach 10-jähriger Beobachtung gesichert sein, um dessen klinischen Einsatz weiterhin rechtfertigen zu können [11]. Aufgrund schlecht verwertbarer kontrollierter Studien und ausreichender Beobachtungs- zeiträume sind viele zementfreie Implantate von dieser Marke weit entfernt.

Im Hinblick auf mögliche Wechseloperationen besteht in Deutschland ein Trend zu zementfreien Verankerungen. Ob die zementfreien oder die zementierten Prothesensysteme bessere Langzeitergebnisse zeigen, ist auch heute noch unter Experten umstritten. Trotz der Fortschritte in der Entwicklung eines besseren Knochenzements und der Optimierung der Zementiertechnik begann man, kontrovers über die Frage zu diskutieren, ob zementierte oder unzementierte Hüftendoprothesensysteme bessere langfristige Ergebnisse in der Alloarthroplastik am Hüftgelenk erzielen. Damit stellt sich die Frage, ob die zementierte Hüftprothese als zweitklassig und veraltet angesehen werden muss und ob man sie nur noch für bescheidene Ansprüche verwenden sollte.
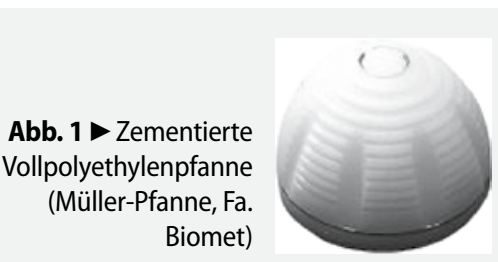

Abb. 2 - Zementierte Schaftprothese (Müller-Geradschaftstiel, Fa. Zimmer)

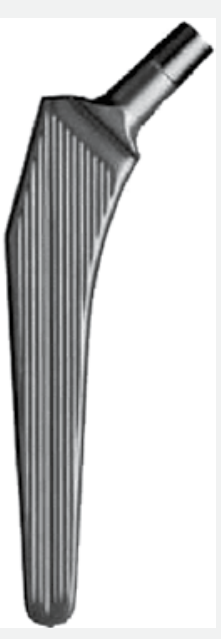




\section{Hüftendoprothetik}

\begin{tabular}{|c|c|c|c|c|c|c|}
\hline Zitat & Jahr & $\begin{array}{l}\text { Pfannen- } \\
\text { system }\end{array}$ & $\begin{array}{l}\text { Fall- } \\
\text { zahl }\end{array}$ & $\begin{array}{l}\text { Follow- } \\
\text { up }\end{array}$ & $\begin{array}{l}\text { Revisions- } \\
\text { rate [\%] }\end{array}$ & $\begin{array}{l}\text { Kumulative Über- } \\
\text { lebensrate [\%] }\end{array}$ \\
\hline $\begin{array}{l}\text { Böhm u. Bösche } \\
\text { [7] }\end{array}$ & 1998 & $\begin{array}{l}\text { Harris- } \\
\text { Galante I }\end{array}$ & 264 & 8 & 1 & 97,7 \\
\hline $\begin{array}{l}\text { Delaunay u. Ka- } \\
\text { pandji }[21,22]\end{array}$ & 1998 & Zweymüller & 382 & 5,0 & 1 & 99 \\
\hline $\begin{array}{l}\text { Effenberger et } \\
\text { al. [24] }\end{array}$ & 1997 & Hofer-Imhof & 136 & 5 & 0,7 & - \\
\hline Fink et al. [31] & 2002 & Bad Bramstedt & 110 & 4,5 & 1,82 & 97,9 \\
\hline Taylor [83] & 2000 & Spotorno (CLS) & 234 & 9 & 2,8 & - \\
\hline
\end{tabular}

\begin{tabular}{|c|c|c|c|c|c|c|}
\hline Zitat & Jahr & $\begin{array}{l}\text { Schaft- } \\
\text { system }\end{array}$ & $\begin{array}{l}\text { Fall- } \\
\text { zahl }\end{array}$ & $\begin{array}{l}\text { Follow- } \\
\text { up }\end{array}$ & $\begin{array}{l}\text { Revisions- } \\
\text { rate [\%] }\end{array}$ & $\begin{array}{l}\text { Kumulative Über- } \\
\text { lebensrate [\%] }\end{array}$ \\
\hline Christie et al. [19] & 1999 & S-ROM & 208 & 4,9 & 0,6 & - \\
\hline Decking [20] & 1999 & ESKA & 96 & 6,2 & 1 & 97 \\
\hline $\begin{array}{l}\text { Delaunay u. Kapandji } \\
{[21,22]}\end{array}$ & 1998 & $\begin{array}{l}\text { Zwey- } \\
\text { müller }\end{array}$ & 238 & 6,5 & 1 & 99,1 \\
\hline Malchau et al. [53] & 2000 & $\begin{array}{l}\text { Spotorno } \\
\text { (CLS) }\end{array}$ & 268 & 7 & 0,6 & - \\
\hline Tonino u. Rahmy [84] & 1999 & $A B G$ & 398 & 5 & 0,8 & - \\
\hline
\end{tabular}

Tab. 3 Langzeitergebnisse verschiedener zementfreier Pfannensysteme

\begin{tabular}{lllllll} 
Zitat & Jahr & $\begin{array}{l}\text { Pfannen- } \\
\text { system }\end{array}$ & $\begin{array}{l}\text { Fall- } \\
\text { zahl }\end{array}$ & $\begin{array}{l}\text { Follow- } \\
\text { up }\end{array}$ & $\begin{array}{l}\text { Revisions- } \\
\text { rate [\%] }\end{array}$ & $\begin{array}{l}\text { Kumulative Über- } \\
\text { lebensrate [\%] }\end{array}$ \\
\hline Epinette et al. [29] & 2003 & Arc2f & 384 & 10 & 0 & 99,4 \\
\hline Hinrichs et al. [38] & 2001 & Marburg & 159 & 10,3 & 5,7 & 92,8 \\
\hline Malchau et al. [52] & 1996 & Lord & 107 & 10 & 37,0 & 44,0 \\
\hline Pospischill u. Knahr [64] & 2005 & $\begin{array}{l}\text { Zwey- } \\
\text { müller }\end{array}$ & 103 & 14,4 & 14,6 & 95,6 \\
\hline Udomkiat et al. [85] & 2002 & APR & 132 & 10,2 & 0,9 & 99,1 \\
\hline
\end{tabular}

Tab. 4 Langzeitergebnisse verschiedener zementfreier Schaftsysteme

\begin{tabular}{llllll} 
Zitat & Jahr & Schaftsystem & Fallzahl & Follow-up & Revisionsrate [\%] \\
Hellmann et al. [37] & 1999 & Omnifit & 67 & 10 & 2,6 \\
\hline Kim et al. [47] & 1999 & AML & 52 & 11,3 & 2 \\
\hline Malchau et al. [53] & 2000 & PCA & 417 & 13 & 27,1 \\
\hline Schramm et al. [73] & 2000 & Spotorno (CLS) & 107 & 10,3 & 0 \\
\hline Zenz et al. [89] & 1995 & Zweymüller & 114 & 10,5 & 3
\end{tabular}

\section{Zementfreie \\ Prothesenimplantation}

\section{Grundlagen}

Um die dauerhafte Verankerung unzementierter Hüftendoprothesen im Knochen zu gewährleisten, ist ein inniger Verbund zwischen Knochen und den eingebrachten Materialien erforderlich. Um dies zu erreichen, ist es erforderlich, die Biomechanik des Hüftgelenks zu erhalten, damit der Knochenstoffwechsel ungestört bleibt und sich somit nach der Einheilungsphase ein biomechanisches Gleich- gewicht an der Knochen-Implantat-Grenze einstellt. Morscher [56] verglich diese Vorgänge mit Prozessen bei der Frakturheilung.

Weiterhin mussten 3 wesentliche Probleme gelöst werden:

- Das Material muss biokompatibel sein und gleichzeitig dem biologisch aggressiven Milieu standhalten, wobei seine Funktionstüchtigkeit, gekennzeichnet durch das gegenseitige $\mathrm{Be}$ wegen und Miteinandergleiten beider Gelenkpartner, erhalten bleiben muss.

- Für beide Gelenkpartner wird eine ausreichende Ausgangsstabilität be- nötigt, auch die Frage der Verankerung muss gelöst werden.

- Die Prothese sollte sowohl einfach zu implantieren als auch - was nicht zu vernachlässigen ist - relativ gut zu explantieren sein.

Zu den Indikationen zur Implantation einer zementfreien Hüftprothese zählen:

- Gute Compliance

- Lange Lebenserwartung

- Junge Patienten

- Gute Knochenqualität

- Gute Schaft- und Pfannengeometrie

- Keine Osteoporose

\section{Osseointegration}

Als Grundvoraussetzung für ein gutes Langzeitergebnis in der zementfreien Hüftendoprothetik gilt die Primärstabilität der Implantate. Sie stellt den entscheidenden Faktor für die postoperative Belastungsfähigkeit des Hüftgelenks dar. Die biologische Aktivität des Knochens muss die Defizite der primären Verankerungsqualität ausgleichen. Eine Überlastung des knöchernen Implantatlagers ist daher in der direkt postoperativen Phase zu vermeiden.

Einen wesentlichen Gesichtspunkt für die dauerhafte Verankerung stellt die sekundäre Osseointegration dar. Osseointegration bedeutet aus histologischer Sicht einen direkten Kontakt zwischen Implantat und Knochen ohne Bindegewebsinterposition [3]. Biomechanisch gesehen muss eine funktionelle Verbindung vom Implantat zum Knochen zur Übertragung der physiologischen Kräfte hergestellt werden. Ein sekundär durch Knocheneinwuchs osteointegriertes und stabilisiertes Implantat schafft die Voraussetzung für eine langfristige Fixation und Funktion ohne Schmerzen oder Lockerung.

Ein großes Problem stellt die dauerhafte biomechanische Integration des Implantats an der Grenzfläche zum lebenden Knochen dar. Aus diesem Grund spielt die Beschaffenheit der Implantatoberfläche eine zentrale Rolle. Sie muss eine gleichmäßige Übertragung der einwirkenden Kräfte gewährleisten und dem Knochen angepasst sein. Die übertragende Fläche sollte möglichst groß sein. Punktförmige 
Kraftübertragungen und damit übergroße lokale Beanspruchungen des Knochengewebes sollten vermieden werden. Diese Forderungen müssen durch entsprechende Wahl der Oberflächenbeschaffenheit und des Designs, d. h. der Formgebung des Implantats und die physikalischen Eigenschaften, erfüllt werden.

Durch die Weiterentwicklung der unzementierten Hüftendoprothesensysteme in den folgenden Jahren kam man den gestellten Anforderungen immer näher. Dies führte zu einer größeren Verbreiterung der zementlosen Technik [86].

\section{Ergebnisse}

Morscher [58] beschrieb für die unzementierte im Vergleich zur zementierten Fixation von Hüftendoprothesen 6 wesentliche Vorteile:

Verbesserung der Knochen-ImplantatGrenze. Es kommt zu keiner Schrumpfung von Knochenzement bei der Polymerisation und keinen Hitzeschäden benachbarter Gewebe. Es entstehen keine Nachteile durch geringe mechanische Widerstandsfähigkeit gegenüber Scher- und Zugkräften sowie große Unterschiede im Elastizitätsmodul zwischen Zement und Implantat bzw. Knochen.

\section{Kleinere Gesamtgröße des Implan-} tats. Durch den Wegfall des Zements verbessern sich die Konservierungsmöglichkeiten.

\section{Erleichterung einer Augmentationsplas-}

tik. Beim Einsatz von Zement können keine Spongiosaplastik durchgeführt oder augmentierte Knochenkeile eingesetzt werden.

\section{Einfaches operationstechnisches Vor- gehen. Im Vergleich zur zementierten Technik sind die Operationszeit kürzer und der Blutverlust geringer, zudem ent- fällt eine mögliche anaphylaktische Reak- tion beim Einbringen des Zements.}

Verbesserte klinische Resultate. Zementfreie Hüftprothesen zeigen in der Regel gute Kurzzeitergebnisse, die mit denen zementierter Prothesen, in Bezug auf
Trauma Berufskrankh 2007 · 9[Suppl 3]: S351-S358 DOI 10.1007/s10039-007-1301-9

(c) Springer Medizin Verlag 2007

\section{S. Özkir $\cdot$ P. Droste $\cdot$ V. Echtermeyer Wann wird zementiert?}

\section{Zusammenfassung}

Ziel des künstlichen Hüftgelenkersatzes sollten die dauerhafte Fixation und die schmerzfreie Funktion der Prothesenkomponenten im Knochen und die Minimierung des Abriebs zwischen den Gleitpartnern sein. Trotz der Optimierung von Knochenzement und Zementiertechnik wird die Frage, ob zementierte oder unzementierte Hüftendoprothesensysteme bessere langfristige Ergebnisse erzielen, kontrovers diskutiert. Es werden die Grundlagen der zementierten und unzementierten Prothesenimplantation, der Operationstechniken sowie der damit verbundenen Ergebnisse und Komplikationen dargestellt. Trotz sämtlicher Vorteile der zementfreien gegenüber der zementierten Prothesenimplantationen zeigen Erstere zwar gute Kurz-

zeitergebnisse, jedoch sind Langzeitergebnisse mit einer Nachuntersuchungszeit von mehr als 10 Jahren nur für eine begrenzte Zahl unzementierter Implantate vorhanden. Somit sollte derzeit, bis Langzeitergebnisse über 15 Jahre für die auf dem Markt befindlichen zementfreien Prothesensysteme vorliegen, die zementierte Verankerung seit Einführung und Anwendung der 2. Generation der Zementiertechniken und der damit einhergehenden Senkung des Revisionsrisikos um etwa $20 \%$, trotz der technisch anspruchsvolleren Implantation, als Standard gelten.

\section{Schlüsselwörter}

Müller-Geradschaft · Hüftendoprothetik .

Zementiertechnik · Fettembolie · Jetlavage

\section{When should joint prostheses be cemented?}

\section{Abstract}

The aims in implantation of an artificial replacement hip joint should be lasting fixation and pain-free functioning of the elements of the prosthesis in the bone and minimal friction between the articulating parts. Despite improvements in bone cement and in cementing techniques, the question of whether cemented or cement-free hip replacement systems give better results in the long term is still the subject of controversy. The basics of cemented and cement-free implantation of prostheses, of the surgical techniques and of the corresponding results and complications are presented. Despite all the advantages of cement-free implantation over cemented implantation of prostheses, while the former yield good results in the short term, long-term results with a follow-up period of over 10 years are available for only a limited number of cement-free implants. Therefore, until long-term (15-year) results for the cement-free prosthetic systems currently on the market are available, cemented anchorage as practised since the introduction and application of the second-generation cementing techniques with the reduction of the risk of revision surgery by approximately $20 \%$ at present should be regarded as standard despite the more demanding implantation process.

\section{Keywords}

Müller straight-stem prosthesis · Hip joint replacement $\cdot$ Cementing technique $\cdot$ Fat embolism · Fluidjet lavage 


\section{Hüftendoprothetik}

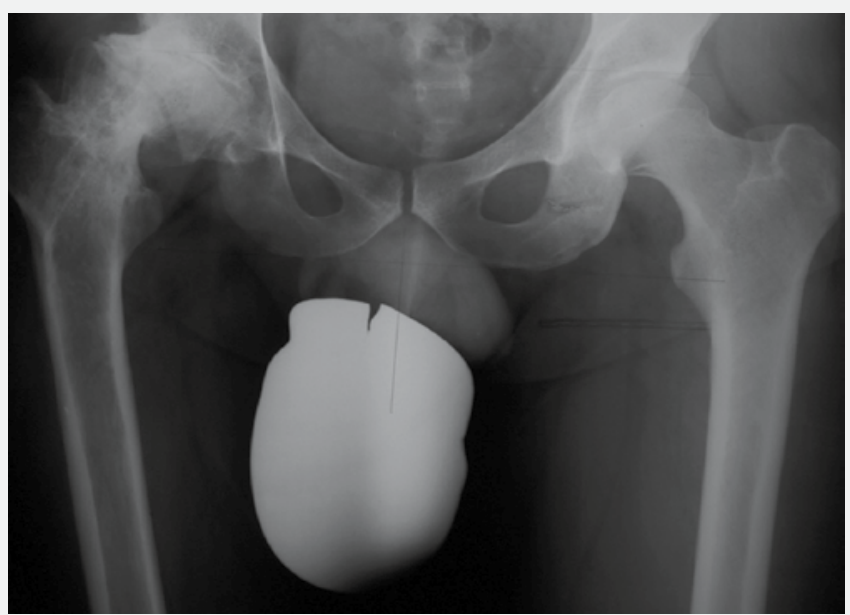

Abb. $3 \Delta$ a.-p. Beckenübersichtsröntgenaufnahme, fortgeschrittene Dysplasiekoxarthrose mit pilzförmiger Deformierung des Femurkopfs und zylindrischer Schaftform im Verlauf des gesamten proximalen Femurs
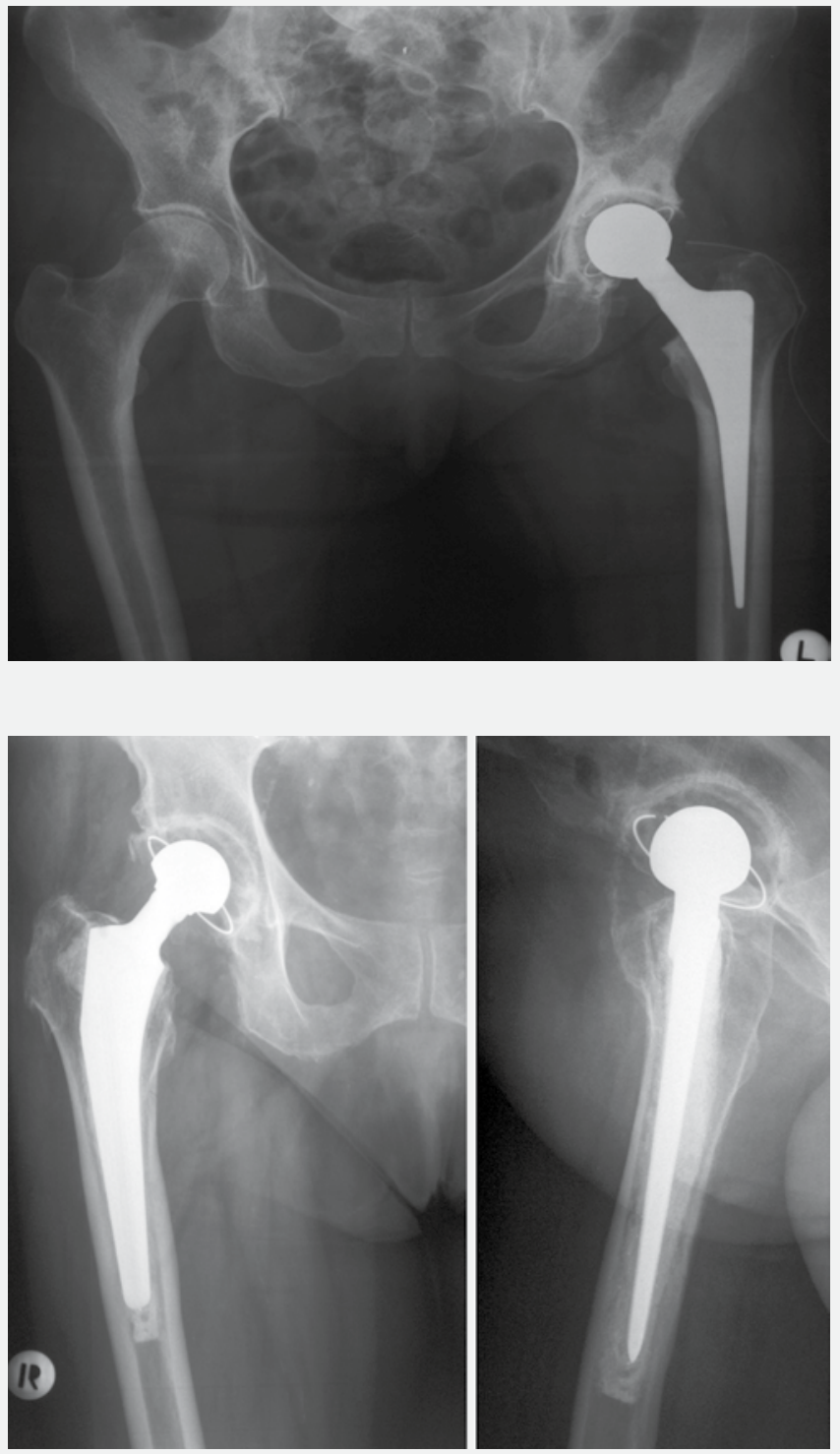

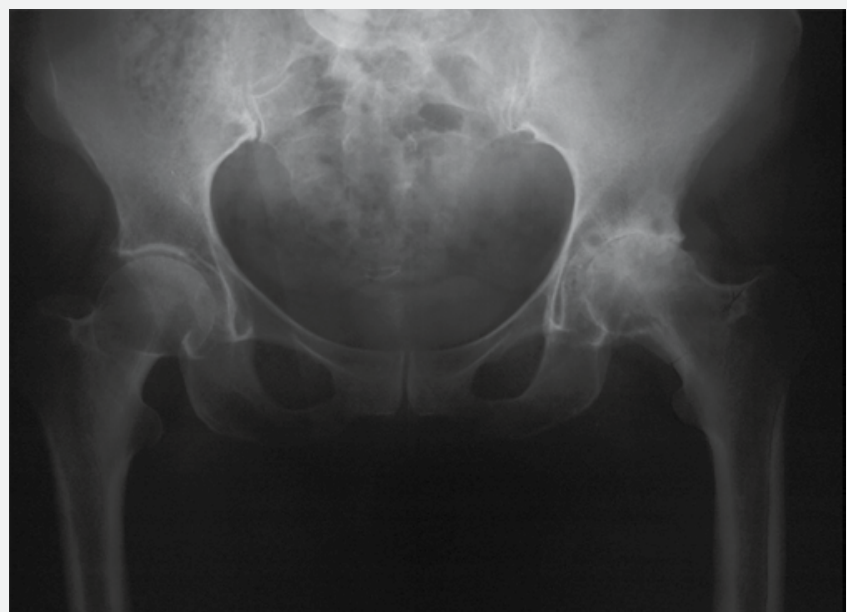

Abb. $4 \Delta$ Röntgenaufnahme, fortgeschrittene Koxarthrose links mit kranialer Abflachung

Abb. $5<$ a.-p. Beckenübersichtsröntgenaufnahme, Zustand nach Totalendoprothesenimplantation am linken Hüftgelenk mit zementierter Vollpolyethylenpfanne (Müller, Fa. Biomet) und zementfreiem Schaft (Spotorno, Fa. Zimmer) postoperativ nach 12 Jahren, regelrechter Implantatsitz mit intaktem Zementmantel an der Pfanne und ohne Osteolysezeichen

Abb. $7<$ Röntgenaufnahme in zwei Ebenen vom 27.07.2007: Postoperativ 17,70 Jahre nach Implantation bei einer zum Nachuntersuchungszeitpunkt 84 jährigen Frau

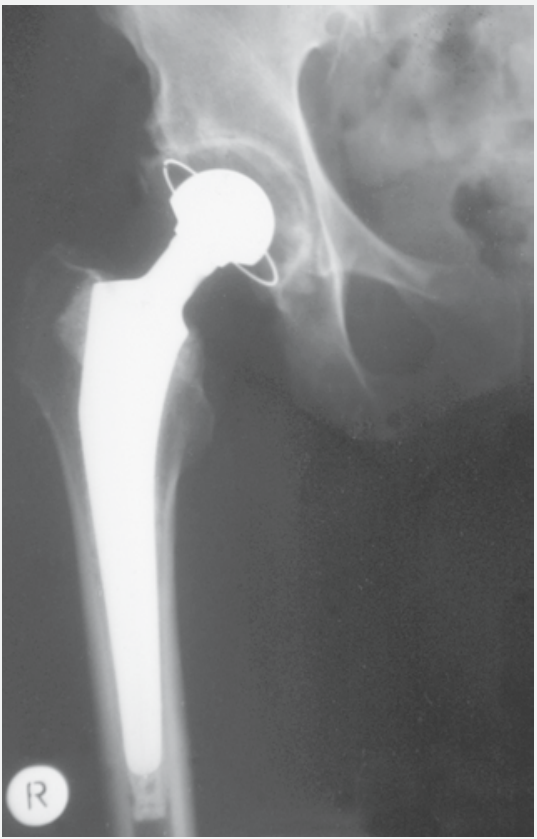

Abb. $6 \Delta$ Postoperative a.-p. Röntgenaufnahme vom 18.11.1989: Totalendoprothesenimplantation am rechten Hüftgelenk mit zementierter Vollpolyethylenpfanne (Müller, Fa. Biomet) und zementiertem Geradschaftstiel (Müller, Fa. Zimmer) 
Schmerzreduktion und Funktion, zu vergleichen sind.

Einfache erfolgreiche Revision. Die Revision bei einer Implantatlockerung ist erleichtert, und es kommt zu weniger Knochenzerstörungen.

Trotz sämtlicher Vorteile der zementfreien gegenüber der zementierten Hüftprothesenimplantation zeigen Erstere in der Regel gute Kurzzeitergebnisse (- Tab. 1, 2), jedoch liegen Langzeitergebnisse mit einem Nachuntersuchungszeitraum von mehr als 10 Jahren nur für eine begrenzte Zahl von unzementierten Pfannen- und Schaftimplantaten vor

(• Tab. 3, 4).

\section{Zementierte \\ Prothesenimplantation}

\section{Grundlagen}

Die zementierte Implantationstechnik stellt heute ein weltweit akzeptiertes Standardverfahren bei der endoprothetischen Versorgung dar. Allein in unserer Klinik wurden im Jahr 2006 von den 273 durchgeführten Hüftendoprothesen 46 Pfannen (Müller-Pfanne, - Abb. 1) und 148 Schäfte (Müller-Geradstiel, - Abb. 2) zementiert. Auch wenn in den deutschsprachigen Ländern und den Vereinigten Staaten (USA) der Anteil der zementfreien Prothesen im Bereich des Schafts deutlich zugenommen hat, ist mit etwa 90\% in Großbritannien und Skandinavien aufgrund der guten Langzeitergebnisse immer noch die zementierte Implantation vorherrschend ( $\bullet$ Abb. 3, 4, 5, 6, 7) [53].

$\mathrm{Zu}$ den Indikationen zur Implantation einer zementierten Hüftprothese zählen:

- Mangelnde Compliance

- Begrenzte Lebenserwartung

- Pathologische SH-Fraktur

- Problematische Pfannen- (Hüftkopfdysplasie) und Schaftgeometrie (Zylinderform)

- Pressfitversagen (Revisionen)

- Osteoporose

\section{Knochenzement (PMMA)}

Mit der Einführung von Polymethylmetacrylat (PMMA) als so genanntem Knochenzement gelang Anfang der 1960er

\section{Tab. 5 Zementiertechniken}

\begin{tabular}{ll} 
Zementiertechnik & Druckspülung (Jetlavage) \\
\cline { 2 - 2 } am Femurschaft & Vakuummischtechnik \\
\cline { 2 - 2 } & Blasenfreie, retrograde Zementapplikation mittels Zementspritze \\
\hline Verwendung eines Markraumstoppers \\
\hline Trockenes Zementbett \\
\hline Zentrierte Implantation \\
\hline Fettembolieprophylaxe \\
\hline $\begin{array}{l}\text { Zementiertechnik } \\
\text { an der Hüftpfanne }\end{array}$ & Haftlöcher im kranialen Pfannendach \\
\hline & Druckspülung (Jetlavage) \\
\hline & Trockenes Zementbett (ggf. $\mathrm{H}_{2} \mathrm{O}_{2}$ ) \\
\hline
\end{tabular}

Tab. 6 Publizierte Ergebnisse der zementierten Müller-Geradschaftprothese

\begin{tabular}{|llllll} 
Zitat & Jahr & Fallzahl & Follow-up & $\begin{array}{l}\text { Revisions- } \\
\text { rate [\%] }\end{array}$ & $\begin{array}{l}\text { Kumulative Über- } \\
\text { lebensrate [\%] }\end{array}$ \\
\hline Acklin et al. [1] & 2001 & 540 & 9,1 & 1,6 & 96,8 \\
\hline Havinga et al. [35] & 2001 & 180 & 10 & 5,9 & 94 \\
\hline Räber et al. [65] & 2001 & 112 & 15 & 7,3 & 92,7 \\
\hline Rader et al. [66] & 2000 & 202 & 6 & 2,5 & 95 \\
\hline Riede et al. [69] & 2007 & 161 & 15 & 11,2 & 94 \\
\hline Schweizer et al. [76] & 2003 & 161 & 10 & - & 98,2 \\
\hline
\end{tabular}

Tab. 7 Publizierte Ergebnisse zementierter Pfannensysteme

\begin{tabular}{|llllll} 
Zitat & Jahr & Pfannensystem & Fallzahl & $\begin{array}{l}\text { Follow- } \\
\text { up }\end{array}$ & $\begin{array}{l}\text { Revisionsrate } \\
{[\%]}\end{array}$ \\
\hline Chen et al. [17] & 1998 & Spectron metalbacked & 86 & 10 & 9,2 \\
\hline Engelbrecht [27] & 1998 & St. Georg/Mark I & 891 & 20 & 18,4 \\
\hline Smith [77] & 2000 & Harris & 47 & 18 & 32 \\
\hline Sochart u. Porter [79] & 1997 & Charnley & 226 & 20 & 27 \\
\hline
\end{tabular}

Tab. 8 Vor- und Nachteile zementierter Hüftendoprothesen

\section{Vorteile}

Ausreichende Primärstabilität

Frühzeitige Mobilisation

Lange Standzeiten

Kostengünstig

Noch immer zeitgemäß

\section{Nachteile}

Erhöhte allergische Reaktion

Erhöhte Wechselproblematik

Lange Aushärtezeiten

Erhöhtes Fettembolierisiko

Anspruchsvollere Operation

Technisch aufwändiger
Jahre Sir Charnley $[14,15]$ aus Großbritannien ein entscheidender Durchbruch in der Hüftendoprothetik.

Methylmetacrylat (MMA) war aus der Zahnheilkunde bekannt, wo es seit 1957 angewendet wurde [56]. Es handelt sich um ein Methylester der Metacrylsäure. Es wird als flüssiges Monomer und als auspolymerisiertes, feinpulveriges Polymer geliefert. Werden Pulver und Flüssigkeit miteinander vermischt, kommt es innerhalb von Minuten zu einer Auspolymerisierung des flüssigen Monomers. Das voll auspolymerisierte Poly-MMA verhält sich gegenüber dem Kontaktgewebe indifferent, allerdings ist das freie Monomer zelltoxisch. Seit Pulver und Flüssigkeit im festen Mischverhältnis geliefert werden, sind die mit der Toxizität des Monomers im Zusammenhang stehenden Komplikationen deutlich zurückgegangen [41, 48].

Abgesehen davon, dass das stets vorgeschriebene Mischverhältnis Monomer: Polymer eingehalten werden muss, ist das Einbringen des Knochenzements von dessen Konsistenz abhängig. Ist der Zement bereits zu hart, gelingt es nicht mehr, ihn in alle im Knochenlager vorbereiteten Vertiefungen und Nuten einzudrücken. Wenn er noch in relativ flüssigem Zustand in den Knochen eingegossen wird, kann es zu einer ungleichmäßigen Verteilung kommen. Infolge unvollständiger oder ungleichmäßiger Verteilung des Zements wird die Übertragung des Kraftflusses von der Totalendoprothese auf das Knochen- 


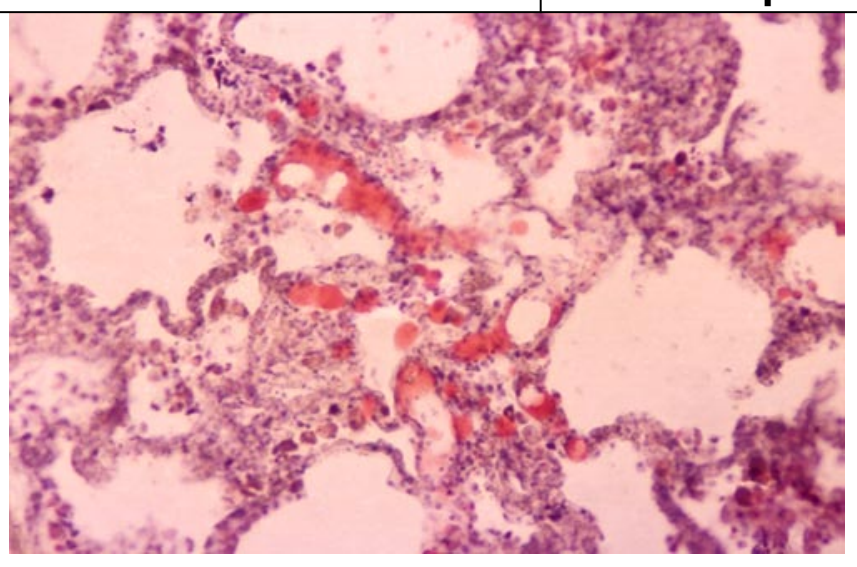

\section{Hüftendoprothetik}

Abb. $8<$ Lungenanschnitt mit Nachweis von Fettembolie (rot) in den peripheren Lungenarterien, Sudan-IIIFärbung, Vergr. 100:1 lager gefährdet. Es entstehen Spannungsspitzen, die zu einem Bruch des Zementköchers oder zur Knochenresorption führen können [32].

Es besteht kein Zweifel, dass durch die Einführung und Anwendung der 2. Generation der Zementiertechniken am Femurschaft mit Druckzementierung, Markraumstopper und Jetlavage zur Spongiosareinigung (• Tab.5) das Revisionsrisiko aufgrund aseptischer Lockerung um etwa $20 \%$ [51] vermindert und damit die Ergebnisse, auch bei jungen Patienten, insgesamt verbessert werden konnten [4, $5,6,13,59,60,70,71,82]$, wobei die pulsierende Druckspülung (Jetlavage) der wichtigste Einflussfaktor war [51]. Moderne Zementiertechniken zielen auf eine bessere Verzahnung des Zements im Knochen, um damit eine erhöhte Scherbeanspruchbarkeit dieser Verbindung zu erreichen [44].

Bis heute jedoch hat sich bei der zementierten Hüftprothesenimplantation besonders die zementierte Pfanne wegen schwer reproduzierbarer Ergebnisse im Langzeitverlauf $\mathrm{z}$. T. als problematisch erwiesen [75, 90]. Die Revisionsraten von zementierten Pfannen liegen häufig deutlich über denen zementierter Schäfte (- Tab. 6, 7).

Charnley [16] hatte in seiner ersten Serie versucht, die kraniale Sklerosezone möglichst vollständig zu entfernen, um eine vermehrte Zementpenetration ins Azetabulum zu erreichen. Bei dieser Präparationsart kam es jedoch in vielen Fällen zur Pfannenwanderung mit ausgeprägten Knochendefekten und notwendigen Wechseleingriffen. Aufgrund dieser Erfahrungen ging Charnley [16] dazu über, die Sklerosezone zu erhalten. Zwar kam es bei diesem Vorgehen zu weniger
Pfannenmigrationen, aber aufgrund einer zu geringen Zementpenetration vermehrt $\mathrm{zu}$ Saumbildungen, insbesondere bei jungen, aktiven Patienten [16]. Zur besseren Verzahnung des Zements wurden multiple Bohrlöcher in die angefrischte, aber erhaltene Sklerose des Pfannendachs eingeführt [25]. Diese noch heute praktizierte Technik der kranialen Verankerungslöcher schuf die Voraussetzung für die weitere Senkung der radiologischen Lockerungsraten [72].

\section{Operationstechnik}

Eine wesentliche Grundlage für akzeptable Langzeitergebnisse in der zementierten Hüftendoprothetik ist eine exakte Operationstechnik, dies schließt die positionsgerechte Implantation der Pfanne und des Schafts ein. Bei optimaler Operationstechnik dringt der Knochenzement (PMMA) in die Hohlräume der offenen Spongiosa ein und begünstigt eine gleichmäßigere Verteilung der Spannungen. Ziel bei der Implantation von zementierten Prothesenkomponenten ist es, einen gleichmäßigen Zementmantel und eine innige Verzahnung von Zement und knöchernem Lager zu erzielen.

Eine geschlossene Zementscheide ist für ein stabiles, belastbares Knochen-Zement-Interface und eine gleichmäßige Kraftübertragung auf das knöcherne Bett erforderlich. $\mathrm{Zu}$ dünne Zementmäntel können zu Defekten und Brüchen führen, die mit erhöhten klinischen und radiologischen Versagerraten verbunden sind $[43,81]$. Des Weiteren führen Implantationsfehlstellungen zu falschen Belastungsmustern, die sich sowohl in atypischen Knochenresorptionen [40] als auch in Alterung des Knochenzements mit massiven
Knochendestruktionen durch Fremdkörpergranulome widerspiegeln können und somit eine frühzeitige Lockerung der Prothese bedingen [33].

\section{Fettembolien}

Aseptische Lockerung, Infektion und Luxation stellen nach wie vor die häufigsten Komplikationen nach Implantation einer Hüfttotalendoprothese dar. Thrombembolische Komplikationen, die das Fettemboliesyndrom mit einschließen, spielen ebenfalls eine nicht zu unterschätzende Rolle. Dies wurde im Rahmen der zementierten Schaftimplantation bereits vor über 30 Jahren erkannt und erforscht. Moderne Zementiertechniken, welche die Anwendung eines Markraumstoppers, einer proximalen Zementkompression und die retrograde und blasenfreien Zementapplikation beinhalten, haben zu einer verbesserten Verzahnung des Zements im Knochen geführt. Bei der Anwendung dieser Verfahren kommt es jedoch zu einer Erhöhung des intramedullären Drucks während der Implantation und damit des Risikos für thrombembolische Komplikationen [36]. Die zementierte Prothesenimplantation scheint dieses Phänomen eher zu begünstigen als die zementfreie Schaftimplantation $[18,39,42]$. Nach Einbringen von Knochenzement (PMMA) bei der Verankerung von Hüfttotalendoprothesen wurden intraoperative Kreislaufkomplikationen in Form von kurzfristigen Blutdruckabfällen und von Herzrhythmusstörungen beschrieben. Sie traten durchschnittlich 2 min nach Einbringen des Knochenzements auf. Diskutiert wurden in diesem Zusammenhang Fettembolien unter Einwirkung von freien Monomeren. Ihre Charakteristika sind:

- Die Formraspel findet kaum Widerstand.

- Der Markbrei ist gelblich ölig.

- Bei der Pfannen- und Schaftimplantation kommt es zu einem Hustenanfall.

Im Tierexperiment konnten die durch freies Monomer verursachten Fettembolien reproduziert werden [23, 74]. Die Verwendung der pulsierenden Druckspülung (Jetlavage) zur Reinigung des knöchernen Lagers erleichtert zum einen die verbes- 
serte Zementpenetration und vermindert zum anderen durch effektive Entfernung von thrombogenen Knochenmarkbestandteilen das Risiko von Fettembolien und kardiorespiratorischen Komplikationen mit potenziell tödlichem Ausgang [12].

Allein in unserer Klinik verstarben im Zeitraum von 1972-1995 bei 4857 implantierten zementierten Hüftprothesen insgesamt 30 Patienten aufgrund einer Fettembolie (• Abb. 8).

\section{Ergebnisse}

In - Tab. 8 sind die Vor- und Nachteile zementierter Hüftendoprothesen aufgeführt.

\section{Fazit für die Praxis}

Trotz sämtlicher Vorteile der zementfreien gegenüber der zementierten Hüftprothesenimplantation zeigen Erstere in der Regel gute Kurzzeitergebnisse, jedoch liegen Langzeitergebnisse mit einem Nachuntersuchungszeitraum von mehr als 10 Jahren nur für eine begrenzte Zahl von unzementierten Pfannen- und Schaftimplantaten vor. Die zementierte Hüftprothesenimplantation sowohl für die Pfanne als auch für den Schaft ist technisch anspruchsvoller als die Implantation einer zementfreien Hüftprothese (• Tab. 8). Jedoch sollte, bis ausschlussreiche Langzeitergebnisse über 15 Jahre für die Vielzahl der auf dem Markt befindlichen zementfreien Prothesensysteme vorliegen, die zementierte Verankerung seit Einführung und Anwendung der 2. Generation der Zementiertechniken mit Druckzementierung, Markraumstopper und Jetlavage zur Spongiosareinigung und deren Senkung des Revisionsrisikos um etwa $20 \%$ momentan als Maß der Dinge gelten.

\section{Korrespondenzadresse}

\section{Dr. S. Özkir}

Unfallchirurgische Klinik, Friedrichstraße 17, 32427 Minden serkan-s.oezkir@klinikum-minden.de

Interessenkonflikt. Der korrespondierende Autor gibt an, dass kein Interessenkonflikt besteht.

\section{Literatur}

1. Acklin YP, Berli BJ, Frick W et al. (2001) Nine-year results of Muller cemented titanium straight stems in total hip replacement. Arch Orthop Trauma Surg 121: 391-398

2. Ahnefelt L, Herberts P, Malchau H et al. (1990) Prognosis of total hip replacement. A Swedish multicenter study of 4.664 revisions. Acta Orthop Scand [Suppl] 238: 1-26

3. Albrektsson T, Branemark PI, Hansson HA et al. (1981) Osseointegrated titanium implants - requirements for ensuring a long-lasting, direct bone-to-implant anchorage in man. Acta Orthop Scand 52: 155-170

4. Ballard WT, Callaghan JJ, Sullivan PM et al. (1994) The results of improved cementing techniques for total hip arthroplasty in patients less than fifty years old. J Bone Joint Surg Am 76: 959-964

5. Barrack RL, Mulroy RD, Harris WH (1992) Improved cementing techniques and femoral component loosening in young patients with hip arthroplasty. J Bone Joint Surg Br 74: 385-389

6. Beckenbaugh RD, Ilstrup DM (1978) Total hip arthroplasty. A review of three hundred and thirtythree cases with long follow-up. J Bone Joint Surg Am 60: 306-313

7. Böhm P, Bösche R (1998) Survival analysis of the Harris-Galante I acetabular cup. J Bone Joint Surg $\mathrm{Br}$ 80: 396-403

8. Bourne RB (1999) The planning and implementation of the Canadian Joint Replacement Registry. Bull Hosp Jt Dis 58: 128-132

9. Bourne RB, Rorabeck CH (1999) Assessing the outcomes: what really works? Orthopedics 22: $823-$ 825

10. Breusch SJ, Aldinger PR, Thomsen M et al. (2000) Verankerungsprinzipien in der Hüftendoprothetik Teil I: Prothesenstiel. Unfallchirurg 11: 918-927

11. Breusch SJ, Aldinger PR, Thomsen M et al. (2000) Verankerungsprinzipien in der Hüftendoprothetik Teil II: Pfannenkomponente. Unfallchirurg 12: 1017-1031

12. Breusch SJ, Reitzel T, Schneider U et al. (2000) Zementierte Hüftendoprothetik - Verminderung des Fettembolierisikos mittels gepulster Druckspülung. Orthopade 29: 578-586

13. Britton AR, Murray DW, Bulstrode CJ et al. (1996) Long-term comparison of Charnley and Stanmore design total hip replacements. J Bone Joint Surg $\mathrm{Br}$ 78: 802-808

14. Charnley J (1960) Anchorage of the femoral head prosthesis to the shaft of the femur. J Bone Joint Surg Br 42: 28-30

15. Charnley J (1970) Total hip replacement by lowfriction arthroplasty. Clin Orthop 72: 7-21

16. Charnley J (1979) Low-friction arthroplasty of the hip. Theory and practice. Springer, Berlin Heidelberg New York

17. Chen FS, Di Cesare PE, Kale AA et al. (1998) Results of cemented metal-backed acetabular components: a 10-year-average follow-up study. J Arthroplasty $13: 867-873$

18. Christie J, Burnett R, Potts HR et al. (1994) Echocardiography of transatrial embolism during cemented and uncemented hemiarthroplasty of the hip. J Bone Joint Surg Br 76: 409-412

19. Christie MJ, DeBoer DK, Trick LW et al. (1999) Primary total hip arthroplasty with use of the modular S-ROM prosthesis. Four to seven-year clinical and radiographic results. J Bone Joint Surg Am 81:1707-1016
20. Decking J, Rosendahl T, Decking D (1999) Proximal fixation of hip endoprostheses with porous surface. Results of 6 years. Z Orthop Ihre Grenzgeb 137: 108-113

21. Delaunay C, Kapandji Al (1998) 10-year survival of Zweymuller total prostheses in primary uncemented arthroplasty of the hip. Rev Chir Orthop Reparat Appar Mot 84: 421-432

22. Delaunay CP, Kapandji Al (1998) Survivorship of rough-surfaced threaded acetabular cups. 382 consecutive primary Zweymuller cups followed for 0.2-12 years. Acta Orthop Scand 69: 379-383

23. Dustmann HO, Schulitz KP, Koch H (1972) Fettembolie nach Anwendung von Knochenzement bei Hüftgelenkersatz. Arch Orthop Unfallchir 72: 114

24. Effenberger H, Weber M, Dorn U et al. (1997) Roentgen criteria and radiologic results of the HoferImhof $(\mathrm{H}-\mathrm{I})$ threaded acetabulum cup in first time implantation. Z Orthop Ihre Grenzgeb 135: 434443

25. Eftekhar NS, Nercessian O (1988) Incidence and mechanism of failure of cemented acetabular component in total hip arthroplasty. Orthop Clin North Am 19: 557-566

26. Ender SA, Machner A, Pap G et al. (2005) Longterm results with the Harris-Galante press-fit-cup. Z Orthop Ihre Grenzgeb 143: 348-354

27. Engelbrecht E (1998) Outcome of the Mark I/II total hip arthroplasty. In: Finerman GAM, Dorey FJ, Grigoris P et al. (eds) Total hip arthroplasty outcomes. Churchill Livingstone, Edinburgh London New York, pp 3-12

28. Engh CA, Bobyn JD, Glassman AH (1987) Porouscoated hip replacement. The factors governing bone ingrowth, stress-shieldings and clinical results. J Bone Joint Surg Br 69: 45-55

29. Epinette JA, Manley MT, D'Antonio JA et al. (2003) A 10-year minimum follow-up of hydroxyapatitecoated threaded cups. J Arthroplasty 18: 140-148

30. Fatz D (2000) Clinica's complete guide to orthopaedics, vol 1. PJB Publications, Richmond

31. Fink $B$, Protzen $M$, Hansen-Algenstaedt $\mathrm{N}$ et al. (2004) High migration rate of two types of threaded acetabular cups. Arch Orthop Trauma Surg 124: 17-25

32. Glien (1994) Materialien der modernen Gelenkendoprothetik. In: Franke J, Graßhoff H (Hrsg) Die zementierte Hüftendoprothese. Buchreihe für Orthopädie und orthopädische Grenzgebiete, Bd 15 Medizinisch Literarische Verlagsgesellschaft $\mathrm{mbH}$, Uelzen

33. Hahn M, Vogel M, Eckstein F et al. (1988) Knochenstrukturveränderungen nach mehrjähriger Hüftgelenksendoprothesenimplantation. Chirurg 59: 782-787

34. Harris WH (1991) Aseptic loosening in total hip arthroplasty secondary to osteolysis induced by wear debris from titanium-alloy modular femoral heads. J Bone Joint Surg Am 73: 470-472

35. Havinga ME, Spruit M, Anderson MA et al. (2001) Results with the M. E. Müller cemented straightstem total hip prosthesis. J Arthroplasty 16: 33-36

36. Heisel C, Mau H, Borchers T et al. (2003) Fettembolie bei der Hüftprothesenimplantation. Zementfrei gegen zementiert - ein quantitativer In Vivo-Vergleich im Tiermodell. Orthopade 32: 247-252

37. Hellman EJ, Capello WN, Feinberg JR (1999) Omnifit cementless total hip arthroplasty. A 10-year average follow-up. Orthopade 364: 164-74

38. Hinrichs F, Boudriot U, Held T et al. (2001) 10-Jahres-Ergebnisse einer Monobloc Hüftendoprothesenpfanne mit mehrlagiger Reintitangitterschale zur zementfreien Implantation. Z Orthop Ihre Grenzgeb 139: 212-217 
39. Hofmann S, Hopf R, Mayr G et al. (1999) In vivo femoral intramedullary pressure during uncemented hip arthroplasty. Clin Orthop 360: 136-146

40. Huiskes R (1993) The current state and future of cemented and non cemented total hip replacement. 1st European Congress of Orthopaedics, Masson. Postgrad Lect 1993: 52-64

41. Hullinger GR (1962) Untersuchungen über die Wirkungen von Kunstharzen in Gewebekulturen. Arch Orthop Unfallchir 54: 581

42. Inadome T, Wall MC, Smith CL et al. (1998-1999) Femoral intramedullary pressure during in vitro cemented and cementless total hip arthroplasty. Orthop Trans 22: 154-155

43. Jasty MJ, Floyd WE, Schiller AL et al. (1986) Localized osteolysis in stable, non septic total hip arthroplasty. J Bone Joint Surg Am 68: 912-919

44. Jasty MJ, Maloney WJ, Bragdon CR et al. (1991) The initiation of failure in cemented femoral components of hip arthroplasties. J Bone Joint Surg Br 73: 551-558

45. Jasty MJ, Bragdon C, Jiranek W et al. (1994) Etiology of osteolysis around porous-coated cementless total hip arthroplasties. Clin Orthop Relat Res 308: 111-126

46. Jasty MJ, Goetz DD, Bragdon CR et al. (1997) Wear of polyethylene acetabular components in total hip arthroplasty. An analysis of one hundred and twenty-eight components retrieved at autopsy or revision operations. J Bone Joint Surg Am 79: 349358

47. Kim YH, Kim JS, Cho SH (1999) Primary total hip arthroplasty with the AML total hip prosthesis. Clin Orthop 360: 147-158

48. König K (1966) Die Restmonomerabgabe bei Kieferorthopädischen Apparaturen aus schnell härtendem Kunststoff. Dtsch Stomatol 16: 816

49. Koseki H, Matsumoto T, Ito S et al. (2005) Analysis of polyethylene particles isolated from periprosthetic tissue of loosened hip arthroplasty and comparison with radiographic appearance. J Orthop Sci 10: 284-290

50. Kreutzer J, Schneider M, Schlegel U et al. (2005) Zementierte Hüftendoprothetik in Deutschland ein Update. Z Orthop Ihre Grenzgeb 143: 48-55

51. Malchau $H$, Herberts $P$ (1998) Prognosis of total hip replacement in Sweden: revision and re-revision rate in THR. 65th Annual Meeting of the American Academy of Orthopaedic Surgeons, February 19-23, New Orleans, USA

52. Malchau H, Herberts P, Wang YX et al. (1996) Longterm clinical and radiological results of the Lord total hip prosthesis. A prospective study. J Bone Joint Surg Br 78: 884-891

53. Malchau $H$, Herberts $P$, Söderman $P$ et al. (2000) Prognosis of total hip replacement. Update and validation from the Swedish National Hip Arthroplasty Registry 1979-1998. 67th Annual Meeting of the American Academy of Orthopaedic Surgeons, 15.-19. March, Orlando, USA

54. Malchau H, Garellick G, Eisler T et al. (2005) The Swedish hip registry. Clin Orthop Rel Res 441: 1929

55. Maronna U (1996) Aktuelle Indikationsstellung zur Teil-/Duokopfprothese. In: Jerosch J, Effenberger H, Fuchs S (Hrsg) Hüftendoprothetik. Thieme, Stuttgart New York

56. Morscher EW (1983) 9 Jahre Erfahrung mit isoelastischen Hüftendoprothesen aus plastischem Material. In: Morscher E (Hrsg) Die zementlose Fixation von Hüftgelenken. Springer, Berlin Heidelberg New York, S 188-195
57. Morscher EW (1987) Experiences, requirements and development of cement free hip endoprostheses. Orthopade 16: 185-196

58. Morscher EW (1995) Die zementfreie Fixation der Hüftgelenkpfanne bei der primären Hüfttotalprothesenarthroplastik. In: Morscher E (Hrsg) Endoprothetik. Springer, Berlin Heidelberg New York

59. Mulroy RD, Harris WH (1990) The effect of improved cementing techniques on component loosening in total hip replacement. An 11-year radiographic review. J Bone Joint Surg Br 72: 757-760

60. Mulroy RD, Harris WH (1997) Acetabular and femoral fixation 15 years after cemented total hip surgery. Clin Orthop 337: 118

61. National Institute of Health (1998) Conventions in total hip arthroplasty. Ministery of Health, London

62. Orsini EC, Byrick RJ, Mullen JB et al. (1987) Cardiopulmonary function and pulmonary microemboli during arthroplasty using cemented or non-cemented components. The role of intramedullary pressure. J Bone Joint Surg Am 69: 822-832

63. Pitto RP, Koessler M, Kuehle JW (1999) Comparison of fixation of the femoral component without cement and fixation with use of a bone-vacuum cementing technique for the prevention of fat embolism during total hip arthroplasty. A prospective, randomized clinical trial. J Bone Joint Surg Am 81: 831-843

64. Pospischill M, Knahr K (2005) Cementless total hip arthroplasty using a threaded cup and rectangular tapered stem. J Bone Joint Surg Br 87: 1210-1215

65. Räber DA, Czaja S, Morscher EW (2001) Fifteenyear results of the Müller $\mathrm{CoCrNiMo} \mathrm{straight} \mathrm{stem.}$ Arch Orthop Trauma Surg 121: 38-42

66. Rader CP, Hendrich C, Low S et al. (2000) [5- to 8year results of total hip endoprosthesis implantation with the Muller straight shaft prosthesis (cemented TiAINb shaft)]. Unfallchirurg 103: 846-852

67. Rahbek O, Kold S, Benedix K et al. (2005) Superior sealing effect of hydroxypatite in porous-coated implants: experimental studies on the migration of polyethylene particles around stable and unstable implants in dogs. Acta Orthop 76: 375-385

68. Revie IC (2000) Fa. Depuy/Johnson \& Johnson, Leeds, England

69. Riede U, Schmid MR, Romero J (2007) Conservative treatment of an acute compartment syndrome of the thigh. Arch Orthop Trauma Surg 127: 269-275

70. Roberts DW, Poss R, Kelley K (1986) Radiographic comparison of cementing techniques in total hip arthroplasty. J Arthroplasty 1: 241-247

71. Russotti GM, Coventry MB, Stauffer RN (1988) Cemented total hip arthroplasty with contemporary techniques. A five-year follow-up study. Clin Orthop 235: 141-147

72. Schneider R (1982) Die Totalendoprothese der Hüfte. Aktuelle Probl Chir Orthop 24: 12-39

73. Schramm M, Keck F, Hohmann D et al. (2000) Total hip arthroplasty using an uncemented femoral component with taper design: outcome at 10-year follow-up. Arch Orthop Trauma Surg 120: 407-412

74. Schulitz KP, Koch H, Dustmann HO (1971) Lebensbedrohliche Sofortkomplikation durch Fettembolie nach Einsetzen von TEP mit Poly-MMA. Arch Orthop Unfallchir 71: 307

75. Schulte KR, Callaghan JJ, Kelley SS et al. (1993) The outcome of Charnley total hip arthroplasty with cement after a minimum twenty-year follow-up. The results of one surgeon. J Bone Joint Surg Am 75: 961-975

76. Schweizer A, Riede U, Maurer TB et al. (2003) Tenyear follow-up primary straight-stem prosthesis (MEM) made of titanium or cobalt chromium alloy. Arch Orthop Trauma Surg 123: 353-356
77. Smith V (2000) Fa. Biomet-Merck, Bridgend, Wales. Zitiert aus Fatz [30]

78. Smith SE, Estok DM, Harris WH (2000) 20-year experience with cemented primary and conversion total hip arthroplasty using so-called second-generation cementing techniques in patients aged 50 years or younger. J Arthroplasty 15: 263-273

79. Sochart DH, Porter ML (1997) The long-term results of Charnley low-friction arthroplasty in young patients who have congenital dislocation, degenerative osteoarthrosis, or rheumatoid arthritis. J Bone Joint Surg Am 79: 1599-1617

80. Spotorno L, Romagnoli S, Ivaldo N et al. (1993) The CLS-System. Theoretical concept and results. Acta Orthop Belg 59: 144-148

81. Star MJ, Colwell CW, Kelmann GJ et al. (1994) Suboptimal (thin) distal cement mantle thickness as a contributory factor in total hip arthroplasty femoral component failure. J Arthroplasty 9: 143-149

82. Stauffer RN (1982) Ten year follow-up study of total hip replacement: with particular reference to roentgenographic loosening of the components. J Bone Joint Surg Am 64: 983-990

83. Taylor JT (2000) 5-13 year results of the Spotorno expansion cup. Acetabulumersatz Meeting, Heidelberg

84. Tonino AJ, Rahmy Al (2000) The hydroxyapatiteABG hip system: 5- to 7-year results from an international multicentre study. The International $A B G$ Study Group. J Arthroplasty 15: 274-282

85. Udomkiat P, Dorr LD, Wan Z (2002) Cementless hemispheric porous-coated sockets implanted with press-fit technique without screws: avarage tenyear follow-up. J Bone Joint Surg Am 84: 11951200

86. Ungethüm M, Blömer W (1987) Technologie der zementlosen Hüftendoprothetik. Orthopade 16: 170-184

87. Willert HG, Bertram H, Buchhorn GH (1990) Osteolysis in alloarthroplasty of the hip. The role of ultra-high molecular weight polyethylene wear particles. Clin Orthop Relat Res 258: 95-107

88. Willert HG, Bertram H, Buchhorn GH (1990) Osteolysis in alloarthroplasty of the hip. The role of bone cement fragmentation. Clin Orthop Relat Res 258: 108-121

89. Zenz P, Pospisil C, Fertschak W et al. (1995) 10 years of cementless implantation of total hip endoprosthesis using Zweymuller's stem. Z Orthop Ihre Grenzgeb 133: 558-561

90. Ziegler BS, Lachiewicz PF (1996) Survivorship analysis of cemented total hip arthroplasty acetabular components implanted with second-generation techniques. J Arthroplasty 11: 750-756 